
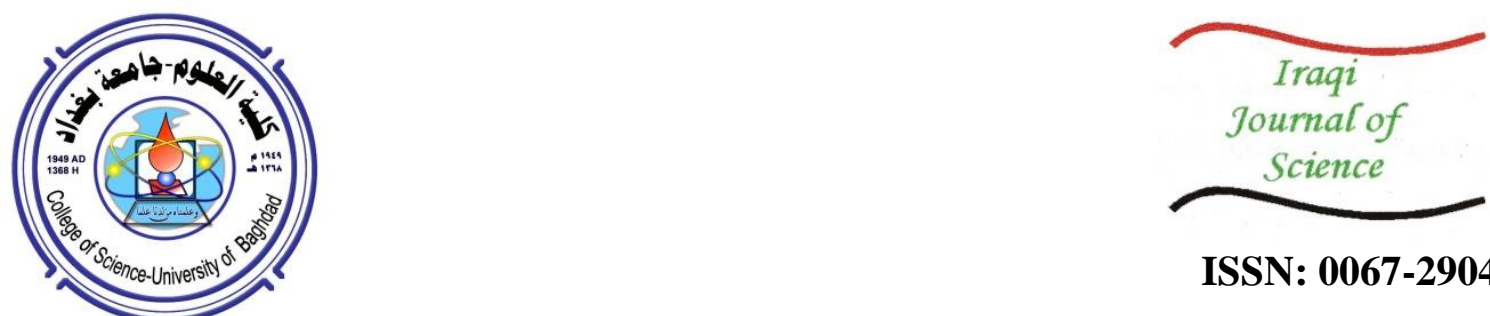

ISSN: 0067-2904

\title{
Epileptic Seizures Detection Using DCT-II and KNN Classifier in Long- Term EEG Signals
}

\author{
Mahmood A. Jumaah ${ }^{1 *}$, Ammar Ibrahim Shihab ${ }^{1}$, Akeel Abdulkareem Farhan ${ }^{2}$ \\ ${ }^{1}$ Department of Computer Science, College of Science, University of Baghdad, Baghdad, Iraq \\ ${ }^{2}$ Department of Computer Science, University of Karbala, Karbala, Iraq
}

\author{
Received: 9/11/2019 Accepted: $17 / 12 / 2019$
}

\begin{abstract}
Epilepsy is one of the most common diseases of the nervous system around the world, affecting all age groups and causing seizures leading to loss of control for a period of time. This study presents a seizure detection algorithm that uses Discrete Cosine Transformation (DCT) type II to transform the signal into frequency-domain and extracts energy features from 16 sub-bands. Also, an automatic channel selection method is proposed to select the best subset among 23 channels based on the maximum variance. Data are segmented into frames of one Second length without overlapping between successive frames. K-Nearest Neighbour (KNN) model is used to detect those frames either to ictal (seizure) or interictal (nonseizure) based on Euclidean distance. The experimental results are tested on 21 patients included in the CHB-MIT dataset. The average F1-score was found to be 93.12, whereas the False-Positive Rate (FPR) average was determined to be 0.07 .
\end{abstract}

Keywords: Epilepsy, EEG, DCT-II, Energy, Seizure Detection, KNN.
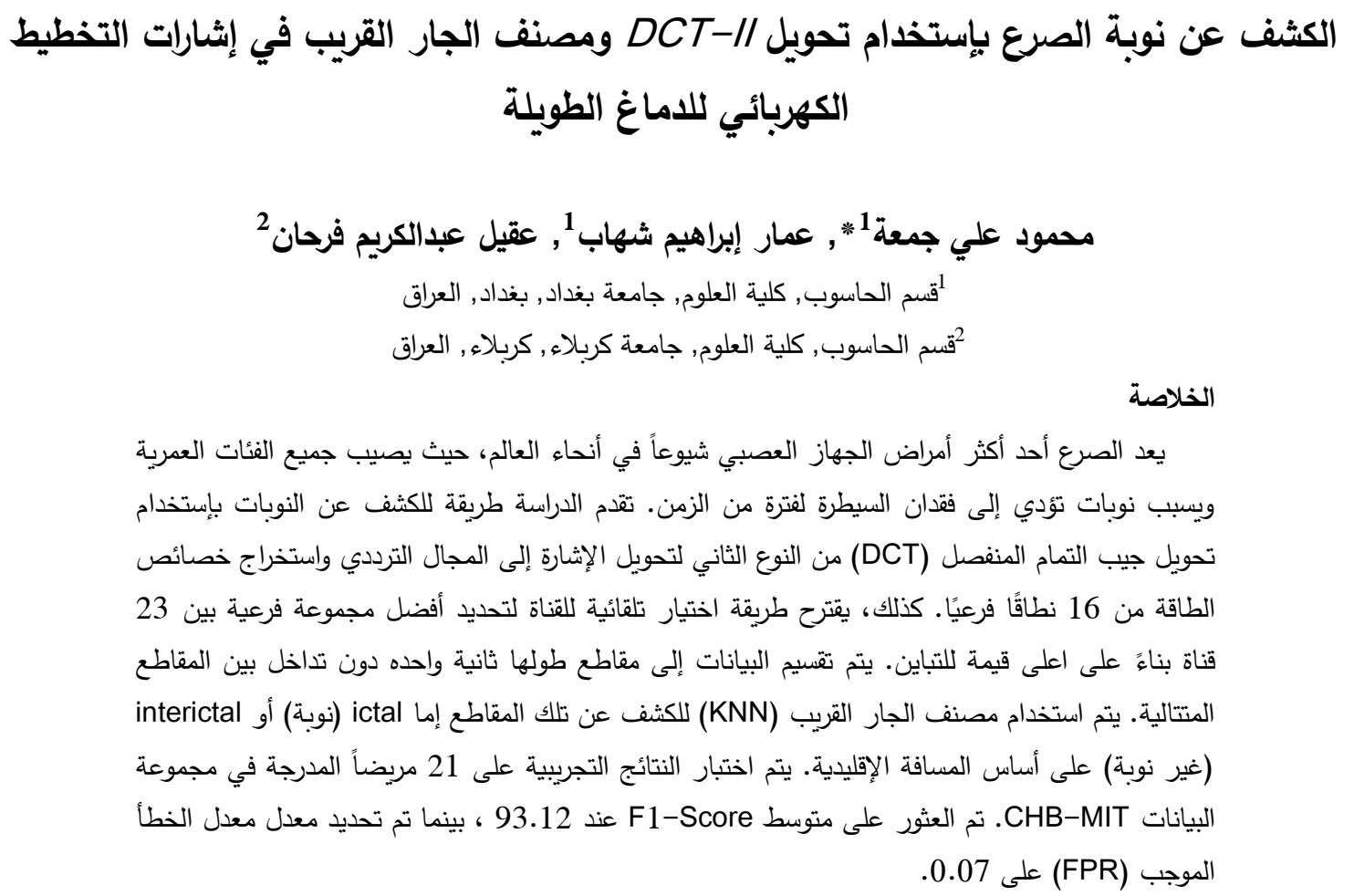

*Email: mahmood.ali2020@yahoo.com 


\section{Introduction}

Epilepsy is one of the widespread disorders and non-communicable disease that affects the human's nerve system [1]. Epileptic seizures cause abnormal behaviours in the electricity of the brain, which produce symptoms such as losing consciousness, tension or convulsion of the whole-body. Patients of epilepsy cannot be aware of the seizure due to differentiation in the nature of human beings, which may increase the physical injury. Moreover, people with epilepsy suffer from social stigma and vocational obstacles. Another load for patients is the condition of continuous seizure activity without a recovery of consciousness between seizures, which is a life-threatening emergency condition [2]. The ability to detect (localize) epileptic seizures would have a deep impact of saving the lives of epilepsy patients [3].

The aim of this paper is to design an algorithm to detect epileptic seizures in epileptic patients using long-term EEG records. The main benefit of the proposed algorithm is using the brain signals recorded by an EEG machine. The proposed algorithm will make it easier for doctors or experts to detect the disease in a fast and accurate way, as it is very difficult to be detected visually by doctors. For example, when a patient lies in the hospital for days (sometimes weeks) while the EEG machine recording the signals; it will be very difficult to check all these signals (long-term EEG) manually. Therefore, an algorithm is proposed to facilitate the method to check these signals automatically while also addressing other problems in the same domain.

The algorithm proposed in this paper is explained by three main stages, as shown in Figure- 1. First, in the pre-processing step, the data were initially loaded, then the most appropriate channels were selected, and signals were epoched into frames to process each of them separately. Second, the features were extracted by using the energy of DCT sub-bands and the best features were selected to be prepared for the classification step. Finally, the KNN model was trained using training set features and the experimental results were evaluated. These steps will be demonstrated in more detail in the methods section.

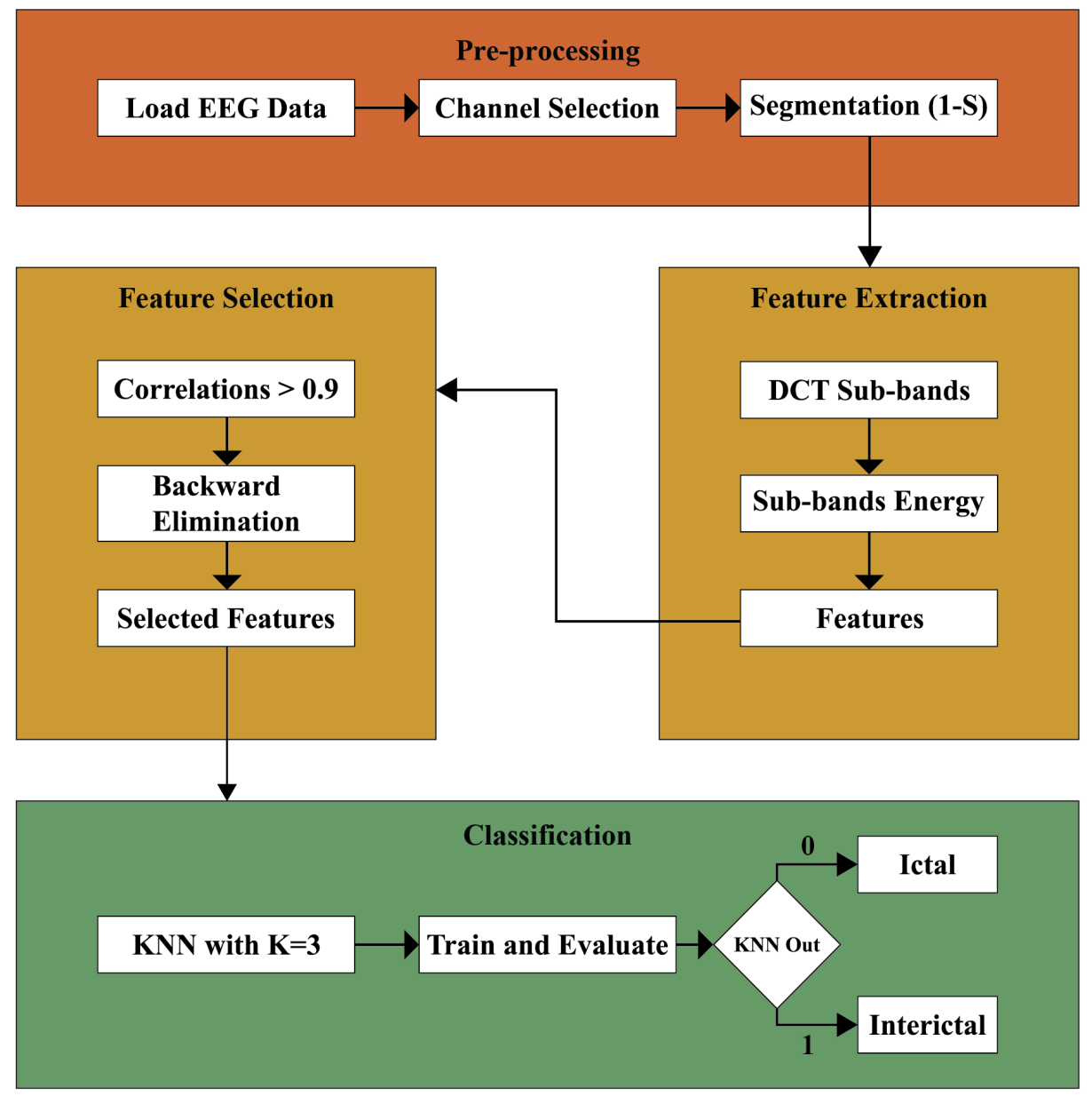

Figure 1-General diagram of the proposed method 


\section{Related Work}

The earliest seizure detection methods were proposed by Gotman in 1976. They rely on the decomposition of EEG records into elementary half-waves. Three features were obtained from epochs of $2 \mathrm{~s}$ : the average amplitude, the average duration of these primary waves, and the variation (ratio of the standard deviation to the mean) of their length as a measure of rhythmicity. Gotman proposed some detection criteria by defining a set of thresholds to classify seizures [4].

Aarabi, in 2007, presented an algorithm to detect seizures in newborn infants using EEG records. This method is composed of feature extraction in several ways which included time-domain, frequency-domain, autoregressive coefficients, and wavelets. Also, EEG classification and pattern recognition were achieved using back-propagation neural network. The average sensitivity of the method was $74 \%[5]$.

Tito, in 2009, showed a nonlinear approach to classify EEG seizure segments by offering nonlinear decision functions with support vector machine (SVM). As a result, the accuracy average was $91.84 \%$ [6].

An automatic seizure detection method was proposed by Yuan in 2012 by using nonlinear features for EEG records to construct the features vector, then feeding them into a single-layer neural network that was trained by extreme learning machine (ELM) algorithm. Finally, the output of the neural network was passed through a post-processing step to make the decision either ictal or interictal. The average sensitivity was approximately $91 \%$ [7].

A novel method was used in 2014 by Yuan to detect seizures automatically by using intracranial EEG. The records were segmented into 4-s window size and decomposed into five levels using wavelet transform. Feature vector was generated using diffusion distances. A total of $193.75 \mathrm{~h}$ were classified using Bayesian linear discriminant analysis (BLDA). The average sensitivity was approximately $94 \%$ [8].

Wang, in 2017, used one-channel EEG record to detect seizure. He showed that it can be transformed into visibility graphs (VGS), including visibility graph (VG), horizontal VG (HVG), and difference VG (DVG). Graphs were used to distinguish various EEG seizure patterns. A total of $615 \mathrm{~h}$ EEG recordings were analysed [9].

A most recent study was proposed by Yan in 2019 to detect seizure using EEG recordings based on image processing techniques. The entire EEG data base was converted into spectrograms. Seizure and non-seizure segments on spectrograms were sequentially sampled as images across a detection window (26380 images). Four Convolutional Neural Networks (CNNs) were trained on these images. The resulting detection performance was suitable for potential clinical use [10].

\section{Materials}

The EEG dataset used in this paper was obtained from Children's Hospital Boston (CHB-MIT) scalp EEG dataset [11], which is public and available at https:// physionet.org/ physiobank/ dataset/ chbmit/. The dataset contains 24 children (males and females) who have epilepsy; each has between 942 continuous EDF (European Data Format) files that contain original raw data of the brain signals. The signals were sampled at 256 samples per second with 16-bit resolution. Most of the dataset files include 23 channels (in some cases 24 or 26 channels). In this study, we selected 21 out of the 24 patients, eliminating the patients 6,12 and 16 because there are some issues to read channels data from the EEG records of those patients. The records were based on electrodes placed according to the International 10-20 system. The dataset contains annotation files for all the 24 cases. For each case, the annotation file includes the number of channels, sample rate, number of seizures, seizure start time, and seizure end time. For more information about the dataset, see Table-1.

\section{Methods}

\subsection{Channel Selection}

The gathered EEG signals are usually of multi-channel recordings. Reducing the number of channels is required because the setup process with a massive number of channels is time-wasting and makes subject inconvenience. Also, it leads to computational complexity. The selection of channels in this study was achieved by calculating the variance of all seizure periods for all channels and then selecting the subset of channels with the highest variance value [12].

$$
\sigma^{2}=\frac{1}{N} \sum_{i=0}^{N-1}\left(x_{i}-\mu\right)^{2}
$$


where $\boldsymbol{x}$ is the samples of signal, $\boldsymbol{\mu}$ is the mean of signal and $\boldsymbol{N}$ is the signal length [12].

Table 1-Description of the CHB-MIT EEG dataset

\begin{tabular}{lcccc}
\hline Subject & Gender & Age & \# of seizure & Duration (hh: mm: ss) \\
\hline \hline chb01 & F & 11 & 7 & $40: 33: 08$ \\
chb02 & M & 11 & 3 & $35: 15: 59$ \\
chb03 & F & 14 & 7 & $38: 00: 06$ \\
chb04 & M & 22 & 4 & $156: 03: 54$ \\
chb05 & F & 7 & 5 & $39: 00: 10$ \\
chb06 & F & 1.5 & 10 & $66: 44: 06$ \\
chb07 & F & 14.5 & 3 & $67: 03: 08$ \\
chb08 & M & 3.5 & 5 & $20: 00: 23$ \\
chb09 & F & 10 & 4 & $67: 52: 18$ \\
chb10 & M & 3 & 7 & $50: 01: 24$ \\
chb11 & F & 12 & 3 & $34: 47: 37$ \\
chb12 & F & 2 & 27 & $20: 41: 40$ \\
chb13 & F & 3 & 12 & $33: 00: 00$ \\
chb14 & F & 9 & 8 & $26: 00: 00$ \\
chb15 & M & 16 & 20 & $40: 00: 36$ \\
chb16 & F & 7 & 10 & $19: 00: 00$ \\
chb17 & F & 12 & 3 & $21: 00: 24$ \\
chb18 & F & 18 & 6 & $35: 38: 05$ \\
chb19 & F & 19 & 3 & $29: 55: 46$ \\
chb20 & F & 6 & 8 & $27: 36: 06$ \\
chb21 & F & 13 & 4 & $32: 49: 49$ \\
chb22 & F & 9 & 3 & $31: 00: 11$ \\
chb23 & F & 6 & 7 & $26: 33: 30$ \\
chb24 & - & $-56: 07$ \\
\hline \hline Total & & & & $\mathbf{9 7 5}$ \\
\hline
\end{tabular}

\subsection{Data Segmentation}

Segmentation means the subdivision of the EEG data into many frames (epochs). In the analysis, the epoching of dataset into segments using specific window-time is taken into account in very necessary pre-processing steps before the feature extraction process. The EEG signals are segmented into different sizes according to the final goal. The window size may be in the range from $0.1 \mathrm{~s}$ to $60 \mathrm{~s}$ [13].

The EEG signals were segmented into 1s frame length with no overlap between successive frames. The latest study by the International Federation of Clinical Neurophysiology (IFCN) [14] proposed that $5 \mathrm{~s}$ is the smallest seizure duration when the background EEG is normal, while it is 10 s when the background EEG is abnormal.

\subsection{Feature Extraction and Selection}

In most modern-day signal processing techniques, frequency-domain transformation and feature extraction have become essential aspects of signal processing. In simple terms, a time-domain signal explains how the real-world signal changes with time [15]. In contrast, a frequency-domain signal shows the rate of variation in signal values and its spectral synthesis.

DCT-II was applied to our dataset recordings to convert signals into frequency-domain. The implementation of DCT-II is simple and performed with low complexity steps that lead to fast transformation, where conversation time takes a few milliseconds only. Lee, in 1984, presented a quick algorithm of DCT-II that has a straightforward recursive structure where the transform splits the signal into even and odd parts [16].

$$
X(n)=\sum_{k=0}^{N-1} x(k) \cos \left\{\pi(2 k+1) \frac{n}{2 N}\right\}
$$


where $\boldsymbol{X}$ is the sample of signal, $\boldsymbol{X}$ is the converted signal, and $N$ is the signal length [16]. The process of configuring the vector features was conducted by passing each frame (Ictal or Interictal) to DCT-II, where the output is divided into 16 sub-bands and the energy is calculated for each band. This process is repeated for each selected channel in the subset. The final feature vector had dimensions of $[\mathrm{F} \times 16 \times \mathrm{N}]$, where $\mathrm{F}$ is the number of frames and $\mathrm{N}$ is the number of selected channels.

$$
\text { Energy }=\frac{1}{N} \sum_{i=0}^{N-1} x_{i}^{2}
$$

where $\boldsymbol{x}$ is the samples of signal and $N$ is the signal length [17].

Covariance matrix is based on the correlation of each feature with all other features [18]. For example, if the number of features equals ten, the matrix dimensions will be [10×10]. Therefore, the feature that has a high correlation (more than 0.9) with the other features must be eliminated in order to increase the classifier accuracy. Finally, the feature selection step was performed by using recursive feature elimination method. This method excludes worst-performing features on an appropriate model one after the other until the best subset of features satisfies [17].

\subsection{Classification}

The most crucial stage in neuroscience applications is the process of classifying brain activity (e.g., EEG signal) into a specific pattern, which can later trusted for decision-making. In machine learning, there are two essential terms that depend on the type of problem and which goal must be achieved; First, Linear models and, second, Non-linear models.

In this study, the KNN model was used. It is a non-linear classifier that is used to classify an unknown point based on the distance between this point and $K$ of its neighbours [19]. Given an unknown feature vector $x$ and a distance measure, then:

- $\quad$ Out of the $N$ training features, the $k$ nearest neighbors are identified, regardless of class label. $k$ is determined to be odd for two-class problems (in this study $K=3$ ).

- Out of these $k$ points, the number of vectors, $k_{i}$, that belong to class $w_{i}, i=1,2, \ldots, M$ is recognized.

$\bullet$

\section{Results} $x$ is put to the class $w_{i}$ with the maximum number $k_{i}$ of points.

CHB-MIT dataset was tested using the DCT-II features by transforming the input signal into the frequency domain. The features used in this stage are the energies of sub-bands for the power spectrum using 16 sub-bands. The KNN classifier classifies each frame either to ictal or interictal in order to finally make the decision. This experimental test seeks to find out how strong this algorithm is with DCT-II features. In this experimental result, five out of 23 channels were used on CHB-MIT dataset. The results in Table-2 show F1-score average of 93.12\% and FPR average of 0.07. These results were obtained using ten-fold cross-validation to ensure that all data were evaluated.

Confusion matrix, also identified as an error matrix, was used for model evaluation. It is a particular table layout that provides visualization of the performance of a classification model, typically a supervised learning. Each record of the matrix describes the cases in a predicted class while each attribute describes the cases in an actual class (or vice versa). The main terminology of confusion matrix are: True Positive (TP) is defined by the count of frames that are correctly classified as ictal, True Negative (TN) is defined by the count of frames that are correctly classified as interictal, False Positive (FP) is determined by the count of frames that are incorrectly classified as ictal and False Negative $(F N)$ are defined by the count of frames are incorrectly classified as interictal.

$$
\begin{aligned}
& \text { Accuracy }=\frac{T P+T N}{T P+T N+F P+F N} \\
& \text { Sensitivity }(T P R)=\frac{T P}{T P+F N} \\
& \text { Specificity }(T N R)=\frac{T N}{T N+F P} \\
& F-\text { Score }=\frac{\left(1+\beta^{2}\right)(P e r c i s i o n * \text { Sensitivity })}{\left(\beta^{2} * \text { Percision }+ \text { Sensitivity }\right)} \\
& \text { FPR }=1-\text { Sensitivity }
\end{aligned}
$$


$F N R=1-$ Specificity

$E R R=1-$ Accuracy

Where $\beta$ can be $0,0.5,1$, or 2 . If $\beta=1$ then the F-Score is called the harmonic mean [18].

Table 2-Results for 21 patients on CHB-MIT dataset using five channels

\begin{tabular}{|c|c|c|c|c|c|c|c|}
\hline Patient & Accuracy & Sensitivity & Specificity & F-Score & FPR & FNR & Error \\
\hline chb01 & 93.86 & 94.24 & 93.12 & 93.69 & 0.07 & 0.06 & 0.06 \\
\hline chb02 & 99.41 & 99.38 & 99.33 & 99.42 & 0.01 & 0.01 & 0.01 \\
\hline chb03 & 99.12 & 100.0 & 98.30 & 99.09 & 0.02 & 0.00 & 0.01 \\
\hline chb04 & 97.38 & 97.06 & 97.33 & 97.34 & 0.03 & 0.03 & 0.03 \\
\hline chb05 & 92.25 & 94.45 & 90.20 & 91.97 & 0.10 & 0.06 & 0.08 \\
\hline chb07 & 94.89 & 95.93 & 93.74 & 94.10 & 0.06 & 0.04 & 0.05 \\
\hline chb08 & 84.32 & 88.52 & 80.86 & 83.25 & 0.19 & 0.11 & 0.16 \\
\hline chb09 & 94.73 & 96.59 & 92.56 & 94.74 & 0.07 & 0.03 & 0.05 \\
\hline chb10 & 96.74 & 99.56 & 94.26 & 96.63 & 0.06 & 0.00 & 0.03 \\
\hline chb11 & 98.32 & 98.29 & 98.39 & 98.33 & 0.02 & 0.02 & 0.02 \\
\hline chb13 & 87.73 & 87.81 & 87.40 & 87.70 & 0.03 & 0.02 & 0.02 \\
\hline chb14 & 86.67 & 90.36 & 83.97 & 85.75 & 0.16 & 0.10 & 0.13 \\
\hline chb15 & 96.76 & 97.99 & 95.52 & 96.69 & 0.04 & 0.02 & 0.03 \\
\hline chb17 & 95.52 & 94.53 & 96.30 & 95.48 & 0.04 & 0.05 & 0.04 \\
\hline chb18 & 93.65 & 95.97 & 91.48 & 93.14 & 0.09 & 0.04 & 0.06 \\
\hline chb19 & 96.81 & 97.41 & 96.34 & 96.64 & 0.04 & 0.03 & 0.03 \\
\hline chb20 & 86.38 & 91.17 & 82.76 & 85.18 & 0.17 & 0.09 & 0.14 \\
\hline chb21 & 85.64 & 82.06 & 88.07 & 84.63 & 0.12 & 0.18 & 0.14 \\
\hline chb22 & 95.75 & 98.97 & 92.65 & 95.60 & 0.07 & 0.01 & 0.04 \\
\hline chb23 & 95.83 & 96.65 & 95.20 & 95.46 & 0.05 & 0.03 & 0.04 \\
\hline chb24 & 90.98 & 93.24 & 88.66 & 90.67 & 0.11 & 0.07 & 0.09 \\
\hline Average & $\mathbf{9 3 . 6 4}$ & $\mathbf{9 4 . 7 7}$ & $\mathbf{9 2 . 2 1}$ & $\mathbf{9 3 . 1 2}$ & $\mathbf{0 . 0 7}$ & $\mathbf{0 . 0 5}$ & $\mathbf{0 . 0 6}$ \\
\hline
\end{tabular}

To compare our results with those from other publications in the same field (seizure detection), five studies were used. Our method proved to be able to detect seizures with a very acceptable accuracy with as few channels as possible compared to the methods shown in Table-3. The methods used in this comparison employed all the channels, and this technique leads to increased complexity, cost, and processing time. Besides, increasing the number of electrodes results in patient discomfort.

Table 3-A comparison with other published works

\begin{tabular}{|c|c|c|c|c|}
\hline Authors & Feature & Classifiers & Dataset & Metrics (\%) \\
\hline Yoo et al [20] & Energy/DFT & SVM & CHB-MIT & Accuracy 84.40\% \\
\hline Dalton et al [21] & Signature & $\begin{array}{c}\text { Template } \\
\text { matching }\end{array}$ & Private & $\begin{array}{c}\text { Specificity } \\
85.00 \%\end{array}$ \\
\hline $\begin{array}{c}\text { Daou and Labeau } \\
{[22]}\end{array}$ & SPIHT codes & Threshold & Bonn & Accuracy 90.00\% \\
\hline $\begin{array}{c}\text { Bajaj and Pachori } \\
{[23]}\end{array}$ & IMF/EMD & Threshold & Freiburg & $\begin{array}{c}\text { Specificity } \\
89.31 \%\end{array}$ \\
\hline Otaiby et al [24] & Histograms & Threshold & CHB-MIT & $\begin{array}{c}\text { Specificity } \\
91.12 \%\end{array}$ \\
\hline Our method & $\begin{array}{c}\text { Energy/DCT- } \\
\text { II }\end{array}$ & KNN & CHB-MIT & $\begin{array}{c}\text { Accuracy } \\
\mathbf{9 3 . 6 4 \%}\end{array}$ \\
\hline
\end{tabular}




\section{Conclusions}

The present study used KNN and DCT-II to detect epileptic seizures in raw EEG recordings using five channels by framing the EEG signals into a 1s long frame, extracting the features using DCT-II and then classifying those features using KNN. A good result was found in relation to the CHB-MIT dataset, and the proposed method can also be used to evaluate very long-term EEG data. As the CHBMIT dataset only includes children, future studies should focus on the EEG data of adults with epilepsy and try to predict seizures using the same method in this research to accomplish a low complexity method to forecast seizures in epileptic patients.

\section{References}

1. Oliva, J.T. and Rosa, J.L.G. 2019. "Classification for eeg report generation and epilepsy detection", Neurocomputing, 335: $81-95$.

2. Aarabi and B. He. 2017. "Seizure prediction in patients with focal hippocampal epilepsy", Clinical Neurophysiology, 128(7): 1299 - 1307.

3. Assi, E.B., Nguyen, D.K., Rihana, S. and Sawan, M. 2017. "Towards accurate prediction of epileptic seizures: A review", Biomedical Signal Processing and Control, 34: 144 - 157.

4. Gotman, J. and Gloor, P. 1976. "Automatic recognition and quantification of interictal epileptic activity in the human scalp eeg”, Electroencephalography and Clinical Neurophysiology, 41(5): 513 $-529$.

5. Aarabi, R. Grebe, and Wallois, F. 2007. "A multistage knowledge-based system for eeg seizure detection in newborn infants", Clinical Neurophysiology, 118(12): 2781 - 2797.

6. Tito, M., Cabrerizo, M., Ayala, M., Barreto, A., Miller, I., Jayakar, P. and Adjouadi, M. 2009. "Classification of electroencephalographic seizure recordings into ictal and interictal files using correlation sum", Computers in Biology and Medicine, 39(7): 604 - 614.

7. Yuan, Q., Zhou, W., Liu, Y. and Wang, J. 2012, "Epileptic seizure detection with linear and nonlinear features", Epilepsy Behavior, 24(4): 415-421.

8. Yuan, S., Zhou, W., Yuan, Q., Zhang, Y. and Meng, Q. 2014, "Automatic seizure detection using diffusion distance and blda in intracranial eeg”, Epilepsy Behavior, 31:339 - 345.

9. Wang, L., Long, X., Arends, J.B. and Aarts, R.M. 2017. "Eeg analysis of seizure patterns using visibility graphs for detection of generalized seizures", Journal of Neuroscience Methods, 290: 85 $-94$.

10. Yan, P.Z., Wang, F., Kwok, N., Allen, B.B., Keros, S. and Grinspan, Z. 2019. "Automated spectrographic seizure detection using convolutional neural networks", Seizure, 2019,

11. Physionet.org. 2019. "CHB-MIT scalp EEG dataset."

12. Alotaiby, T., El-Samie, F.E.A., Alshebeili, S.A. and Ahmad, I. 2015. "A review of channel selection algorithms for eeg signal processing”, EURASIP Journal on Advances in Signal Processing, 2015: 66.

13. Alba, J., Marroquín, L., ArceSantana, E. and T. Harmony, T. 2010. "Classification and interactive segmentation of eeg synchrony patterns", Pattern Recognition, 43(2): 530 - 544.

14. Weerd, D. 2000. "Chapter 36 eeg in neonates. what does the neonatal eeg tell about prognosis?", in Clinical Neurophysiology at the Beginning of the 21st Century (Z. Ambler, S. Nevšmalová, Z. Kadaňka, and P. Rossini, eds.), vol. 53 of Supplements to Clinical Neurophysiology, pp. 243 -249, Elsevier.

15. Chatterjee, R., Maitra, T., Islam, S.H., Hassan, M.M., Alamri, A. and Fortino. 2019, "A novel machine learning based feature selection for motor imagery eeg signal classification in internet of medical things environment", Future Generation Computer Systems, 98: 419 - 434.

16. Byeong Lee. 1984. "Fct -a fact cosine transform", in ICASSP '84. IEEE International Conference on Acoustics, Speech, and Signal Processing, 9: 477-480.

17. Mursalin, M., Zhang, Y., Chen, Y. and Chawla, N. 2017. "Automated epileptic seizure detection 
using improved correlation-based feature selection with random forest classifier", Neurocomputing, vol. 241.

18. Yang, G., Liu, Y. and Pan, G. 2019. "Weighted covariance matrix estimation", Computational Statistics Data Analysis, 139: 82 - 98, 2019.

19. Theodoridis, S. and Koutroumbas, K. 2009, "Chapter 4 - nonlinear classifiers", in Pattern Recognition (Fourth Edition) (S. Theodoridis and K. Koutroumbas, eds.), pp. 151 - 260, Boston: Academic Press, fourth edition ed.

20. Yoo, J., L. Yan, El-Damak, D., Altaf, M.A.B., Shoeb, A.H. and Chandrakasan, A.P. 2013. “An 8channel scalable eeg acquisition soc with patient-specific seizure classification and recording processor", IEEE Journal of Solid-State Circuits, 48: 214-228.

21. Dalton, S. Patel, A. Chowdhury, M. Welsh, T. Pang, S. Schachter, G. Ó Laighin, and P. Bonato. 2012. "Development of a body sensor network to detect motor patterns of epileptic seizures", IEEE transactions on biomedical engineering, 59: 3204-11.

22. Daou, H. and Labeau, F. 2014, "Dynamic dictionary for combined eeg compression and seizure detection", IEEE journal of biomedical and health informatics, 18: 247-256.

23. Bajaj, V. and Pachori, R. 2013. "Epileptic seizure detection based on the instantaneous area of analytic intrinsic mode functions of eeg signals", Biomedical Engineering Letters, 03: 17-21.

24. Otaiby, T., Alshebeili, S.A., Alotaibi, F.M. and Alrshoud, S.R. 2017. "Epileptic seizure prediction using csp and lda for scalp eeg signals", Computational Intelligence and Neuroscience, 2017: 111. 